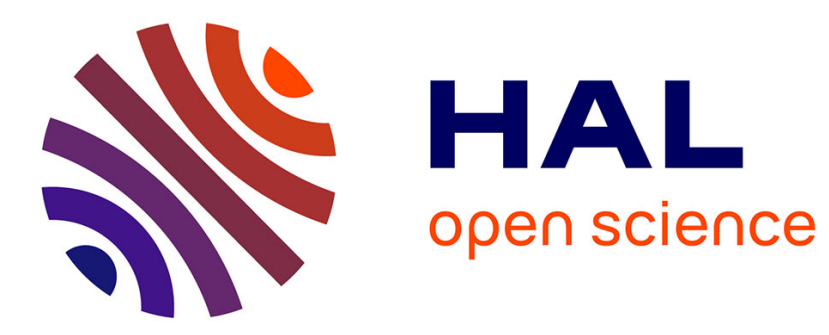

\title{
On the Spectral Characteristics of Ad Hoc Networks and their Mobility Properties
}

\author{
Alonso Silva, Gabriel Tucci
}

\section{To cite this version:}

Alonso Silva, Gabriel Tucci. On the Spectral Characteristics of Ad Hoc Networks and their Mobility Properties. International Conference on Mobile Ad-Hoc and Sensor Networks, Dec 2011, Beijing, China. pp.215 - 222, 10.1109/MSN.2011.52 . hal-01076515

\section{HAL Id: hal-01076515 https://inria.hal.science/hal-01076515}

Submitted on 22 Oct 2014

HAL is a multi-disciplinary open access archive for the deposit and dissemination of scientific research documents, whether they are published or not. The documents may come from teaching and research institutions in France or abroad, or from public or private research centers.
L'archive ouverte pluridisciplinaire HAL, est destinée au dépôt et à la diffusion de documents scientifiques de niveau recherche, publiés ou non, émanant des établissements d'enseignement et de recherche français ou étrangers, des laboratoires publics ou privés. 


\title{
On the Spectral Characteristics of Ad-hoc Networks and their Mobility Properties
}

\author{
Alonso Silva, Gabriel H. Tucci ${ }^{\dagger}$
}

October 26, 2011

\begin{abstract}
Random geometric graphs have proven to be extremely useful in modeling static wireless ad-hoc and sensor networks. The study of these graphs and their spectral properties is a very active field with many interesting applications. In this work, we study the spectral properties of these graphs and in particular we prove the lack of spectral gap in random geometric graphs under a wide range of cases. We also present some results on the spectral properties of generalized weighted versions of these graphs. We later focus on mobile geometric graphs or dynamic Boolean models as were introduced by van den Berg, Meester, and White in 1997. These mobile models have been used to model mobile ad-hoc and sensor networks. Our results are aimed at understanding the dynamics of message spreading in a realworld dynamical network. We simulate a message spreading process and present some results related to the total propagation time under different scenarios. We call total propagation time the time it takes for a certain message to reach all the nodes in the network. This message could be a packet, a virus, a rumor in a social network, or something more general. In particular, we study how the metric, mobility properties, presence or absence of obstacles and finally the propagation characteristics affect the dynamics of the message spreading.
\end{abstract}

*UC Berkeley, CA, USA. Email: asilva@eecs.berkeley.edu

${ }^{\dagger}$ Bell Laboratories, NJ, USA. Email: gabriel.tucci@alcatel-lucent.com 


\section{Introduction and Motivation}

A random geometric graph consists of a set of vertices distributed randomly over some metric space, with two vertices joined by an edge if the distance between them is sufficiently small. More precisely, we consider the following model: let $I=[0,1]^{d}$ the $d$-dimensional unit cube and let $G\left(n, r_{n}\right)$ be the graph formed when $n$ nodes are placed uniformly and independently in $I$, and two nodes are connected if the distance between them is less than $r_{n}$. The radius $r_{n}$ is referred to as the range of a node. Many of the properties of these graphs have been studied and analyzed, including the connectivity properties, cover times and mixing times (see $[1,4,7,9,10,14,15]$ ). For instance, it was proved in $[4,7,14]$ that the graph is connected almost surely if and only if

$$
r_{n} \geq\left[\frac{1}{n}\left(\frac{2(d-1)}{d} \log (n)+\frac{2}{d} \log \log (n)+\gamma(n)\right)\right]^{1 / d}
$$

for any sequence $\gamma(n) \rightarrow \infty$. The study of random geometric graphs is a relatively new area; the monograph [14] is a great reference. In addition to their theoretical interest, random geometric graphs have many applications, including wireless communication networks and ad-hoc networks. While simple, these models capture important qualitative features of real world ad-hoc networks.

Human trajectories are often approximated by different models of random walks. Measurements suggest that animal but also human trajectories can be approximated by Lévy flights $[17,18,19,20]$. Investigating mobility at the geographical scale, is relevant for individual mobility and proximity in contexts that are relevant for data diffusion and their applications. A wide range of commonly used models can be found in the survey about mobility models and ad-hoc networks by Camp et al. [21]. The authors emphasize the need to devise accurate mobility models, and explore the limitations of current modeling strategies, pointing out that models with no memory (random walk) describe nodes whose actions are independent from one another. On the other hand, group mobility models, such as the Nomadic Community Mobility Model, aim at representing the behavior of nodes as they move together. 
Most analytical frameworks for message diffusion (see, e.g., [22]) are stochastic models used to compute message delay distributions based on parameters describing communication range and inter-contact time distributions, with no special characterization of the causal structure of message propagation. Other works such as [23] and [24] also focus on the analysis of the distributions of inter-meeting intervals. Another relevant area deals with modeling data dissemination in opportunistic networks. In [25] data is pro-actively disseminated using a strategy based on the utility of the data itself. Utility is defined on top of existing social relationships between users, and the resulting Markovian model is validated in simulation.

In this work we study the mobility properties in random geometric graphs. In particular, we study the propagation time and how it is affected by changes in the metric and the topology of the geographical area. We further explore how the propagation time is affected by the characteristics of the information spread. In other words, how different models for the information spread affect the propagation time.

This paper is organized as follows. In Section 2, we prove that for any sequence $r_{n} \rightarrow 0$ these graphs have no spectral gap as $n \rightarrow \infty$. More precisely, given the normalized Laplacian of $G\left(n, r_{n}\right)$ (to be defined later), this one is a positive definite operator with eigenvalues $0=\mu_{1} \leq \mu_{2} \leq \ldots \mu_{n} \leq 2$ we prove that $\mu_{2} \rightarrow 0$ almost surely as $n \rightarrow \infty$. We also compute the spectral measure under different regimes. In Section 3, we study a generalized version of the random geometric graph where the points are randomly placed in a $d$-dimensional compact manifold $\mathcal{M}$ and where the links between nodes have certain weights. We prove that when the weights are defined from a Gaussian weighting kernel then the normalized Laplacian of the graph converges towards the continuous Laplace-Beltrami operator of the manifold as the number on nodes increases. In Section 4, we continue the study done in [16] and [10] regarding the mobility properties of these graphs and how fast information spreads. Our main interest is in studying the propagation time. How long does it takes for a disease to spread to an entire population? How does it change as we change the topology, the radius of coverage, the metric, the mobility characteristics and the propagation characteristics? We first consider the Euclidean and hyperbolic metric. In the Euclidean case the nodes are located in the unit square or torus with the Euclidean metric and they move independently of the other nodes accordingly to a standard 
Brownian motion or a Lévy flight of exponent $\mu$. In the hyperbolic case the nodes are located in the disk $\mathbb{D}(0, R)$ inside the hyperbolic space with the Poincaré disk model. Regarding the propagation characteristic we consider two scenarios. In the first one we start by one infected node and every node becomes infected after being in contact with an infected node. The second scenario is called the bootstrap model and a node becomes infected only after being in contacted with two or more different infected nodes but not necessarily at the same time.

\section{Lack of Spectral Gap}

\subsection{Background and Notation}

Let $G=(V, E)$ be a graph with vertex set $V$ and edge set $E$. Let $d_{v}$ denote the degree ${ }^{1}$ of a vertex $v$. The normalized Laplacian of the graph $G$ is defined to be the operator

$$
\mathcal{L}(u, v)=\left\{\begin{aligned}
1 & \text { if } u=v \text { and } d_{v} \neq 0 \\
\frac{-1}{\sqrt{d_{u} d_{v}}} & \text { if } u \text { and } v \text { are adjacent } \\
0 & \text { otherwise }
\end{aligned}\right.
$$

The operator $\mathcal{L}$ can be seen as an operator on the space of functions over the vertex set $V$. If the graph $G$ is infinite and locally finite (every vertex has finite degree), then $\mathcal{L}$ is a well defined operator from $l_{2}(V)$ to itself ${ }^{2}$. In other words, $\mathcal{L}: l_{2}(V) \rightarrow l_{2}(V)$ where for each $x \in l_{2}(V)$ we define $\mathcal{L} x$ as

$$
\mathcal{L} x(u)=\frac{1}{\sqrt{d_{u}}} \sum_{v \sim u}\left(\frac{x(u)}{\sqrt{d_{u}}}-\frac{x(v)}{\sqrt{d_{v}}}\right)=x(u)-\sum_{v \sim u} \frac{x(v)}{\sqrt{d_{u} d_{v}}} .
$$

It is well known (see for instance [2]) that the operator $\mathcal{L}$ is positive definite and its spectrum is contained in the interval [0,2]. Moreover, if the graph is finite then the element 0 always belongs to the spectrum. This is not the case for infinite graphs; we say that an infinite graph has a spectral gap if

\footnotetext{
${ }^{1}$ The degree of a vertex is defined as the number of edges incident to the vertex.

${ }^{2}$ The space $l_{2}(V)$ is the space of square-summable sequences defined over $V$.
} 
the element 0 does not belong to the spectrum of $\mathcal{L}$. Since the spectrum of $\mathcal{L}$ is compact, this is equivalent to saying that the bottom of the spectrum $\lambda_{0}$ defined as

$$
\lambda_{0}(G)=\inf \{\lambda \in \operatorname{spec}(\mathcal{L})\}=\inf \left\{\|\mathcal{L} x\|_{2}:\|x\|_{2}=1\right\}
$$

is strictly positive. If the graph $G$ is finite then $\lambda_{0}(G)$ is defined as the value of the second eigenvalue. In other words, if $|G|=n$ then the Laplacian operator has eigenvalues $0=\mu_{1} \leq \mu_{2} \leq \ldots \leq \mu_{n} \leq 2$, and the bottom of the spectrum is defined as $\lambda_{0}(G):=\mu_{2}$.

Another important property of a graph is its Cheeger constant. Firstly, consider a graph $G$ and $S$ a subset of the vertices of $G$, we define $\operatorname{vol}(S)$, the volume of $S$, to be the sum of the degrees of the vertices in $S$ :

$$
\operatorname{vol}(S)=\sum_{x \in S} d_{x}
$$

for $S \subset V(G)$. Secondly, we define the edge boundary $\partial S$ of $S$ to consist of all the edges with one endpoint in $S$ and the other in the complement $S^{c}=V \backslash S$. The Cheeger constant of an infinite graph is defined as

$$
h_{G}=\inf \left\{\frac{|\partial S|}{\operatorname{vol}(S)}: S \subset V(G) \text { with }|S|<\infty\right\} .
$$

It is a clear consequence of this definition that strictly positive Cheeger constant implies linear isoperimetric inequality, since for every finite subset $S$ of $G$ we have that $|\partial S| \geq h_{G} \cdot \operatorname{vol}(S)$. The following well-known result $[2,12,13]$ relates the value of the Cheeger constant with the second eigenvalue $\mu_{2}$,

$$
2 h_{G} \geq \mu_{2} \geq 1-\sqrt{1-h_{G}^{2}}
$$

for any graph $G$, where the second inequality assumes that $G$ is connected.

Example 2.1. For the infinite linear chain, the integers $\mathbb{Z}$, the spectrum is equal to the whole interval [0,2]. Moreover, for every $p \geq 2$ the spectrum of $\operatorname{spec}\left(\mathbb{Z}^{p}\right)=[0,2]$.

Example 2.2. Let $k \geq 3$ and let $G$ be the $k$-regular tree also known as the $k$-Bethe lattice. It is known (see for example [11, 12]) that

$$
\operatorname{spec}\left(T_{k}\right)=\left[1-\frac{2 \sqrt{k-1}}{k}, 1+\frac{2 \sqrt{k-1}}{k}\right] .
$$


Therefore, the larger the degree $k$ is, the smaller the spectrum becomes. Moreover, the spectral measure for the normalized Laplacian is given by

$$
\mu(d x)=\frac{\sqrt{4(k-1)-k^{2}(1-x)^{2}}}{2 \pi k\left(1-(1-x)^{2}\right)} \cdot \mathbf{1}_{\left[1-\frac{2 \sqrt{k-1}}{k}, 1+\frac{2 \sqrt{k-1}}{k}\right]} .
$$

This shows in particular that for the k-regular tree the spectral gap for such a tree is equal to $1-\frac{2 \sqrt{k-1}}{k}$.

\subsection{Lack of Spectral Gap for the Random Geometric Graphs}

In this section we will prove the lack of spectral gap for the random geometric graphs in a very general context. Assume without loss of generality that $n$ nodes are deployed randomly in the unit $d$-dimensional cube $I=[0,1]^{d}$ and any two nodes are connected if their Euclidean distance is less than $r_{n}$. We are interested in the spectral properties of this graph as $n$ goes to infinity. We would like to mention that all our arguments work if we replace the unit cube by a finite volume, open and connected domain in $\mathbb{R}^{d}$. The parameters $n$ and $r_{n}$ determine a graph that we will denote $G\left(n, r_{n}\right)$.

Theorem 2.3. For any sequence $r_{n} \rightarrow 0$ then the second eigenvalue $\mu_{2}(n)$ of $G\left(n, r_{n}\right)$ goes to zero almost surely.

Proof. For notation simplicity let us focus in the case $d=2$, all the arguments can be carried out for the other cases. The interesting case is when the graph is connected since otherwise $\mu_{2}=0$. Therefore, let us assume that $r_{n}$ is greater than the connectivity threshold that in the two-dimensional case is known to be equal to

$$
r_{n} \geq r_{\text {con }}=\left(\frac{\log (n)+\gamma(n)}{n \pi}\right)^{\frac{1}{2}}
$$

where $\gamma(n)$ is any sequence such that $\gamma(n) \rightarrow \infty$. Under this assumption we know that the second eigenvalue $\mu_{2}(n)>0$ and we will show that $\mu_{2}(n) \rightarrow 0$ almost surely as $n \rightarrow \infty$. For every node $x$ it is clear that the expected degree $\mathbb{E}(\operatorname{deg}(x))=n \pi r_{n}^{2}$. 
Consider $X$ the set of nodes in $\left[0, \frac{1}{2}\right] \times[0,1]$ and $Y$ the set of nodes in $\left[\frac{1}{2}, 1\right] \times$ $[0,1]$, without loss of generality assume that $|X| \leq \frac{n}{2}$, moreover $|X| \rightarrow \frac{n}{2}$ almost surely as $n \rightarrow \infty$. Let $\epsilon>0$ and define $T_{\epsilon}$ the set of nodes in $\left[\frac{1}{2}-\epsilon, \frac{1}{2}\right] \times[0,1]$. Since $r_{n} \rightarrow 0$ there exists $n$ sufficiently large such that all the nodes in $X \backslash T_{\epsilon}$ are not connected to the nodes in $Y$.

Let $h_{n}$ the Cheeger constant of $G\left(n, r_{n}\right)$. Then by definition

$$
h_{n} \leq \frac{|\partial X|}{\operatorname{vol}(X)} .
$$

Now $\operatorname{vol}(X) \rightarrow \frac{n}{2} \mathbb{E}(\operatorname{deg}(x))$ almost surely and $|\partial X| \leq \operatorname{area}\left(T_{\epsilon}\right) n \mathbb{E}(\operatorname{deg}(x))$. Therefore,

$$
h_{n} \leq 2 \operatorname{area}\left(T_{\epsilon}\right)=2 \epsilon .
$$

Since $\epsilon$ is arbitrary and independent on $n$ we see that $h_{n} \rightarrow 0$ almost surely. Now using Equation (6) we conclude the proof.

\subsection{Simulation Results}

In this section we present some simulation results regarding the spectral measure of random geometric graphs and the rate of decay of $\mu_{2}$ as $n$ increases.

In Figure 1 we see the spectral measure for $G\left(n, \sqrt{\frac{\log (n)}{\pi n}}\right)$ for $n=1000$ and 10 trials. In Figure 1 we see the value of $\mu_{2}$ the second eigenvalue of the Laplacian as a function of $n$.

\section{Spectral Properties of Generalized Random Geometric Graphs}

Random geometric graphs can be generalized in many different ways. One possibility is instead of randomly deploy the nodes in a finite volume domain in $\mathbb{R}^{d}$ is to deploy them in a finite volume $d$-dimensional Riemannian manifold $\mathcal{M}$ and connect the nodes if the geodesic distance between the nodes is smaller than a threshold $r$. This procedure gives us a graph embedded in the manifold $\mathcal{M}$. Another possible generalization is to assign weights to 

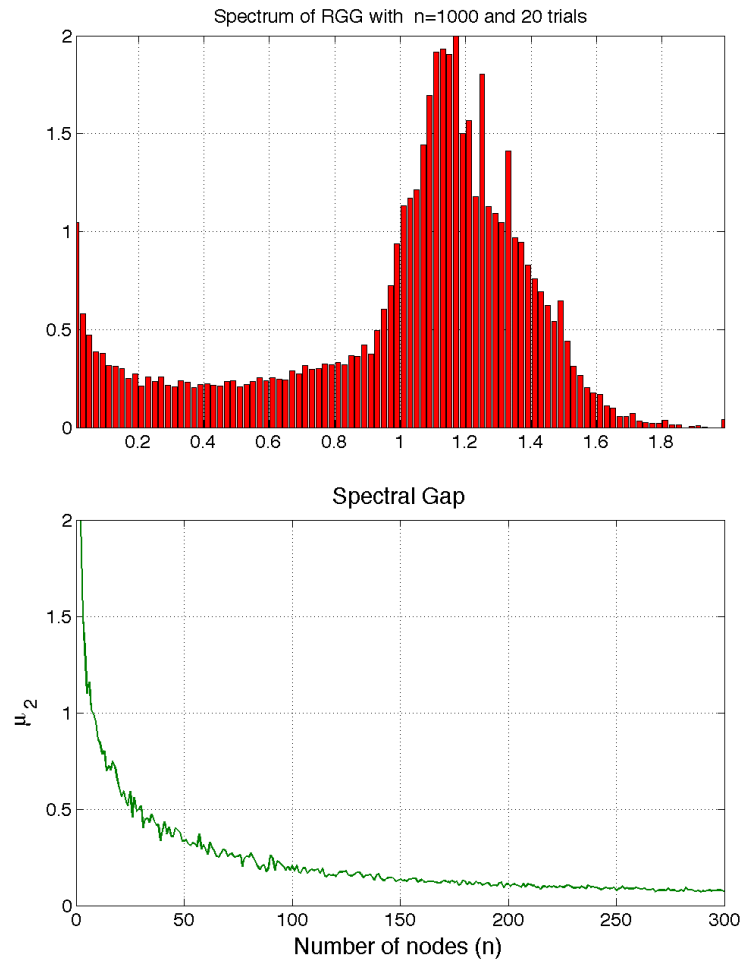

Figure 1: The top chart is the spectral density of $G\left(n, \sqrt{\frac{\log (n)}{\pi n}}\right)$ for $n=1000$ and 10 trials. The bottom chart is the second eigenvalue $\mu_{2}(n)$ as a function of $n$. 
the different links. Note that in the classical random geometric graphs all the links have weight one. This may not be a reasonable assumption if we are modeling ad-hoc networks since the signal strength changes continuously with the distance between the nodes.

In this section we would like to focus in the spectral properties of this generalized model. Our model consists of a $d$-dimensional manifold $\mathcal{M}$, a weight function $f: \mathbb{R}^{+} \rightarrow \mathbb{R}^{+}$and we will deploy $n$ nodes uniformly random accordingly to the volume measure induced by the Riemannian metric in $\mathcal{M}$. Given any two nodes $u$ and $v$ the weight of their link will be given by $w(u, v)=f(d(u, v))$ where $d(u, v)$ is the distance between the nodes. A reasonable weight function to consider would be

$$
w(u, v)=\exp \left(-d(u, v)^{2} / 2 \sigma\right)
$$

for $\sigma>0$. We will denote this graph as $G_{\mathcal{M}}(n, w)$. Note that if $f(x)=$ $\mathbf{1}_{[0, r]}(x)$ we recover the classical random geometric graph in $\mathcal{M}$. For a weighted graph $G=(V, E)$ the Laplacian operator $\mathcal{L}: l_{2}(V) \rightarrow l_{2}(V)$ is defined as in Equation 3 but with the according weights. In other words, for each $x \in l_{2}(V)$ we define $\mathcal{L} x$ as

$$
\mathcal{L} x(u)=x(u)-\sum_{v \sim u} \frac{w(u, v) x(v)}{\sqrt{d_{u} d_{v}}} .
$$

Given a manifold $\mathcal{M}$ there is a natural differential operator called the LaplaceBeltrami operator. This one is defined as the divergence of the gradient, and is a linear operator taking functions into functions. The operator can be extended to operate on tensors as the divergence of the covariant derivative. Alternatively, the operator can be generalized to operate on differential forms using the divergence and exterior derivative (for more on this see [3]). Combining the definitions of the gradient and divergence, the formula for the Laplace-Beltrami operator applied to a scalar function $f$ is, in local coordinates

$$
\mathcal{L}_{\mathcal{M}} f=\operatorname{div} \operatorname{grad} f=\frac{1}{\sqrt{|g|}} \sum_{i=1}^{d} \sum_{j=1}^{d} \partial_{i}\left(\sqrt{|g|} g^{i j} \partial_{j} f\right) .
$$

Here $|g|:=\left|\operatorname{det}\left(g_{i j}\right)\right|$ is the absolute value of the determinant of the metric tensor $g_{i j}$. The divergence $\operatorname{div} X$ of a vector field $X$ on the manifold in local 
coordinates is equal to

$$
\operatorname{div} X=\frac{1}{\sqrt{|g|}} \sum_{i=1}^{d} \partial_{i}\left(\sqrt{|g|} X^{i}\right)
$$

The gradient of a scalar function $f$ is the vector field $\operatorname{grad} f$ that may be defined through the inner product $\langle\cdot, \cdot\rangle$ on the manifold, as

$$
\left\langle\operatorname{grad} f(x), v_{x}\right\rangle=d f(x)\left(v_{x}\right)
$$

for all vectors $v_{x}$ anchored at point $x$ in the tangent space $T_{x} \mathcal{M}$ of the manifold at point $x$. Here, $d f$ is the differential of the function $f$. In local coordinates, one has

$$
(\operatorname{grad} f)^{i}=\partial^{i} f=\sum_{j=1}^{d} g^{i j} \partial_{j} f
$$

where $g^{i j}$ are the components of the inverse of the metric tensor, so that $g^{i j} g_{j k}=\delta_{i k}$ with $\delta_{i k}$ the Kronecker delta.

Many examples of the Laplace-Beltrami operator can be worked out explicitly.

Example 3.1 (Euclidean space). In the usual (orthonormal) Cartesian coordinates $x^{i}$ on the Euclidean space, the metric is reduced to the Kronecker delta, and one therefore has $|g|=1$. Consequently, in this case

$$
\mathcal{L} f=\frac{1}{\sqrt{|g|}} \sum_{i=1}^{d} \sum_{j=1}^{d} \partial_{i} \sqrt{|g|} \partial^{i} f=\sum_{i=1}^{d} \sum_{j=1}^{d} \partial_{i} \partial^{i} f
$$

which is the ordinary Laplacian. In curvilinear coordinates, such as spherical or cylindrical coordinates, one obtains alternative expressions.

Example 3.2 (Spherical Laplacian). The spherical Laplacian is the LaplaceBeltrami operator on the $(d-1)$-sphere with its canonical metric of constant sectional curvature 1. It is convenient to regard the sphere as isometrically embedded into $\mathbb{R}^{d}$ as the unit sphere centred at the origin. Then for a function $f$ on $S^{d-1}$, the spherical Laplacian is defined by

$$
\mathcal{L}_{S^{d-1}} f(x)=\mathcal{L} f(x /|x|)
$$


where $f(x /|x|)$ is the degree zero homogeneous extension of the function $f$ to $\mathbb{R}^{d}-0$, and $\mathcal{L}$ is the Laplacian of the ambient Euclidean space. Concretely, this is implied by the well-known formula for the Euclidean Laplacian in spherical polar coordinates:

$$
\mathcal{L} f=r^{1-n} \frac{\partial}{\partial r}\left(r^{n-1} \frac{\partial f}{\partial r}\right)+r^{-2} \mathcal{L}_{S^{n-1}} f
$$

Now we are ready to prove the main theorem of this section.

Theorem 3.3. Let $G_{\mathcal{M}}(n, w)$ with weight function as in Equation 11. Let $\mathcal{L}$ be the Laplace operator of the graph $G_{\mathcal{M}}(n, w)$ and let $\mathcal{L}_{\mathcal{M}}$ be the LaplaceBeltrami operator of $\mathcal{M}$. Then for any differentiable function $f: \mathcal{M} \rightarrow \mathbb{R}$ we have that

$$
\lim _{n \rightarrow \infty} \mathcal{L} f(u)=\frac{\sigma}{2} \mathcal{L}_{\mathcal{M}} f(u)+O\left(\sigma^{3 / 2}\right) .
$$

Similar results were proved in [26] using different techniques. Our proof is considerably shorter but we will omit it in this paper.

\section{Mobility Random Geometric Graph Model}

In many situations, the nodes of a graph are not fixed but mobile. Many different types of mobility can be considered for the nodes but it seems natural to allow some room for randomness in the mobility properties. These models can be used to understand properties of communication networks, social networks, ad-hoc networks, sensor networks and also the spread of infectious diseases and rumors. Even though these models are simple they capture important characteristics of real networks. In [16] the problems of detection (time until a target node is detected), coverage (the time until all nodes in a large set are detected) and percolation (the time until a given node is connected to an infinite connected component) were analysed. Their model consists of an infinite number of nodes deployed randomly according to a Poisson point process on $\mathbb{R}^{d}$ of intensity $\lambda$. Each node $X_{i}$ moves according to a standard Brownian motion $\left\{\xi_{i}(t)\right\}_{t \geq 0}$ independently of the other nodes. Therefore, at any given time $t$ we have the graph $G_{t}$ constructed by

adding an edge between any two nodes that are at distance at most $r$. As 
it is mentioned in [16], the analysis where the number of nodes is infinite and nodes are deployed in a infinite volume space like $\mathbb{R}^{d}$ is mathematically cleaner and explicit formulas can be proved since we do not have boundary effects.

In this work we choose to work in the finite volume case with a finite number of nodes. This scenario is more realistic from a practical point of view. We consider a multi-hop network with $n$ mobile nodes with radius of coverage $r_{n}$. We suppose that the radius of coverage is the same for every node and we adopt the geometric graph model, i.e., two nodes are connected or they can exchange information if the distance between them is inferior to a certain threshold (called radius of coverage), otherwise, they are disconnected. This model can also be used to study the spread of infectious diseases or rumors. More specifically, starting from one or several infected nodes we can study how fast the infection spread to the whole population and how this change as we change the mobility properties of the nodes, their velocity, the radius of coverage and the metric.

As we mentioned in the introduction our main interest is in studying the propagation time and how this is affected by the changes in the radius of coverage, the metric, the mobility characteristics and the propagation characteristics.

\subsection{Description of the Code}

The code can basically be decomposed in four programs: 'fixBoundaries', 'infect', 'simulate', and 'monteCarlo'.

The program 'fixBoundaries' enforces the boundaries of the domain. It takes as parameters the position of the nodes, the direction of the nodes, and the domain description. The program detects which nodes are about to escape from the domain and depending on the domain description, it bounces and handles them accordingly.

The program 'infect' detects which nodes are infected and which nodes are not, and depending on the position of the nodes and the radius of coverage, it infects the nodes that are within the radius of coverage of an infected node. It takes as parameters the current infection of the nodes, the position of the 
nodes, and the radius of coverage.

The main program 'simulate' computes the propagation time over the nodes. It takes as parameters the number of nodes, the radius of coverage, the speed of the nodes, and time between direction changes. The program chooses the position of the nodes uniformly at random over the considered domain. The direction of the nodes is chosen uniformly at random and the speed is a gaussian variable with mean and standard deviation equal to the speed of the nodes. It infects one of the nodes at random. Then at each time, it computes the nodes that are going to be infected by using the program 'infect' and the new positions of the nodes according to the mobility model considered and enforcing the boundaries by using the program 'fixBoundaries'. When all the nodes have been infected, it terminates and gives the propagation time.

The program 'monteCarlo' uses Monte Carlo methods to compute the propagation time by using the main program.

These programs are available upon request.

\subsection{Propagation Time on the Euclidean Space}

In this subsection, we consider the Euclidean distance as the metric of the space, i.e., the distance between two points $\left(x_{1}, y_{1}\right)$ and $\left(x_{2}, y_{2}\right)$ is given by

$$
d_{E}\left(\left(x_{1}, y_{1}\right),\left(x_{2}, y_{2}\right)\right)=\sqrt{\left(x_{1}-x_{2}\right)^{2}+\left(y_{1}-y_{2}\right)^{2}} .
$$

The values of the parameters we have used in our simulations are listed as follows:

1. The network area is the two-dimensional unit square $I=[0,1] \times[0,1]$ (where nodes bounce against the boundary in a billiard like fashion). We also study the two dimensional torus $\mathbb{T}$.

2. The number of mobile nodes in the network is $n=500$.

3. Nodes start from a uniformly distributed initial position.

4. Each node takes a uniformly distributed direction angle in $[0,2 \pi)$. 
5. We evaluated different scenarios where all mobile nodes change their direction after $T_{1}=1 \mathrm{~s}, T_{2}=5 \mathrm{~s}, T_{3}=10 \mathrm{~s}$.

6. Each mobile node changes its speed at each new time interval and choose its speed at random with a Gaussian distribution $N\left(0, v^{2}\right)$ where $v=0.005$ diam $/ \mathrm{s}$. Therefore, the standard deviation is $0.5 \%$ of the diameter of the whole domain per second. This mobility mimics a Brownian motion.

7. All the simulations are averaged over 50 instances to obtain an estimate of the average.

8. We take at random one mobile node and consider it infected.

9. We assume that all of the nodes within range of an infected node will became infected.

The simulation shows the total propagation time, i.e., the time it takes for all of the nodes to be infected, as a function of the range. Note that as the time $T$ becomes smaller and smaller the mobility of the nodes gets closer and closer to a Brownian motion. We did not observe any significant change by changing the space from $[0,1]^{2}$ to the torus $\mathbb{T}$, so it seems that the boundary effect is not that relevant in the propagation time. Figure 2 shows the propagation time as a function of the coverage radius. To make comparisons easier when we change the metric we always plot propagation time versus radius of coverage normalised by the diameter of the domain over 2 .

\subsection{Lévy Flights}

As in the previous subsection we consider the Euclidean distance to be the metric of the space. All the simulation parameters are as before but we change the mobility properties of the nodes. They still move independently of each other but instead of moving accordingly to a Brownian motion they move accordingly to the so called Lévy flight. Recently, many empirical studies and theoretical analysis on the pattern of animal foraging, migration and human travelling have presented that these mobility patterns possess a Lévy flights property with an exponent $\mu=-1.59$ for humans. More 

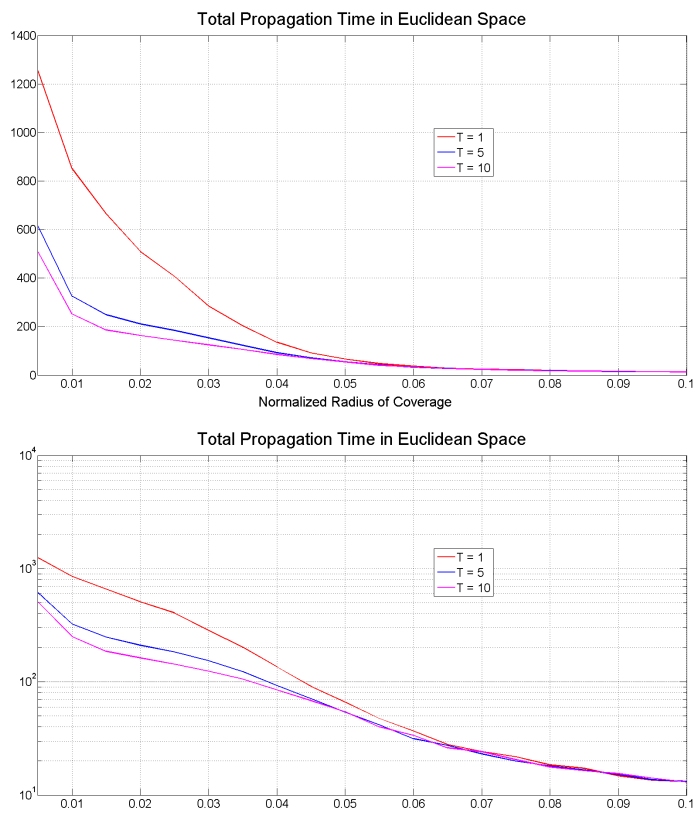

Figure 2: Total propagation time as a function of the radius of coverage normalized by the diameter for three different mobility scenarios in the Euclidean space. The bottom figure is the log scale of the top one. 
precisely, if a node at time $t$ is in position $(x(t), y(t))$ then his next position will be

$$
\begin{aligned}
& x(t+1)=x(t)+r \cos (2 \pi \theta) \\
& y(t+1)=y(t)+r \sin (2 \pi \theta)
\end{aligned}
$$

where $\theta$ is random and uniformly distributed on $[0,1]$ and $r$ is a random variable with power law distribution of parameter $\mu$. More precisely, its probability distribution is given by

$$
p(r)=\mu r_{0}^{\mu} \frac{1}{r^{1+\mu}} \cdot \mathbf{1}_{\left[r_{0}, \infty\right)}(r) .
$$

The value $r_{0}$ is the cut-off parameter that we fix it at $r_{0}=0.003$. In Figure 3 we can appreciate the differences between Brownian diffusion and the Lévy flight diffusion with various exponents. The Lévy flight type moving pattern of individuals has many local steps but the occasional long distance journeys. The smaller the parameter $\mu$ the more frequent this long journeys occur. This has consequences for epidemic models of disease spreading in physical space. In Figure 4 we compare the total propagation time for different exponents of the Lévy flights and Brownian motion. We can appreciate that the spread of information is much faster when nodes are allowed to travel long distances in short periods of time as modelled in the Lévy flight case.

\subsection{Bootstrap Propagation on the Euclidean Space}

As in the previous subsection, we consider the Euclidean distance to be the metric of the space. This model is a little bit more realistic for the spread of a disease or a rumor. A node does only became infected after being in contact with 2 or more different infected nodes (not necessarily at the same time). For rumors this capture the idea that if somebody tells you a rumor you don't necessarily believe it right away but if several people tell you the same rumor you are more likely to believe it.

The values of the parameters we have used in our simulations are as follows:

The first (1)-(7) are the same as in the previous simulation.

8. A node become infected after being in contact with two infected nodes. 

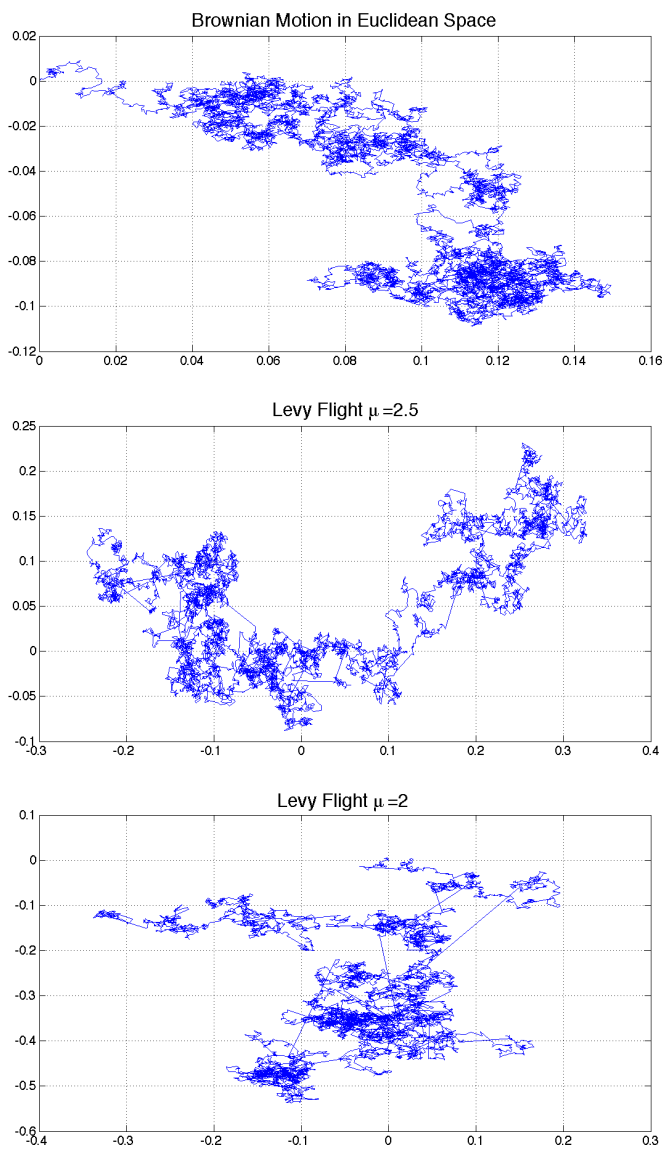

Figure 3: On top we can see a node describing a Brownian motion in the unit square $[0,1]^{2}$. In the middle we can see a node performing a Lévy flight with $\mu=2.5$. In the bottom figure we have a node performing a Lévy flight with parameter $\mu=2$. In the three cases the node is performing 15, 000 steps. 

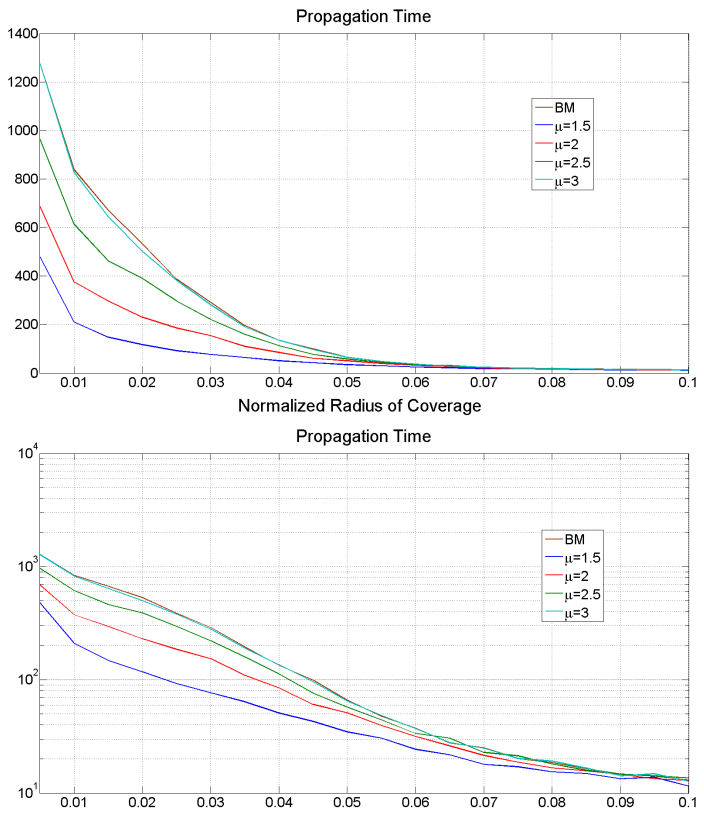

Figure 4: Total propagation time for Brownian motion movements and Lévy flights. The number of nodes is 500 in the unit square. The bottom figure is the log plot of the top one. 


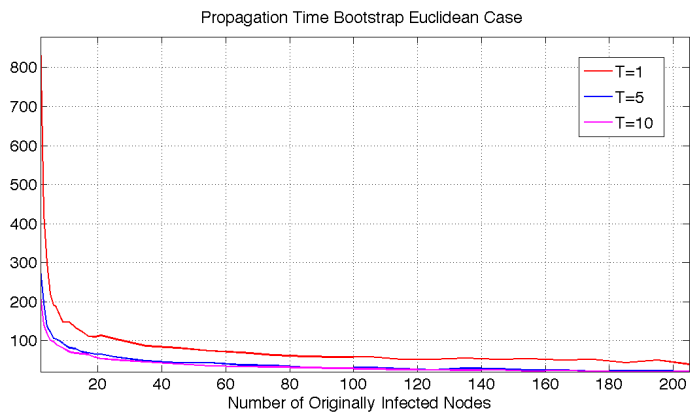

Figure 5: Total propagation time as a function of the initial number of infected nodes in the bootstrap case with the Euclidean metric.

9. We choose at random $M$ mobile nodes and infect them.

10. We assume that all of the nodes within range of an infected node will be infected as well.

11. We consider a radius of coverage equal to $r=0.05 \cdot \operatorname{diam} / 2$.

The simulation in Figure 5 shows the total propagation time as a function of the initial number of infected nodes $M$ for $T=1 s, 5 s$ and $10 \mathrm{~s}$. We can appreciate a sharp transition in the propagation time around $M=4$. In the case $M=1$ the disease never spreads since we need at least two infected nodes, for $M$ between 2 and 4 we can appreciate a very fast decay in the propagation time form $M$ greater than 5 the decay is much less pronounced. This behavior was somehow expected for us and can be heuristically interpreted as the existence of threshold than when this number is reached the disease spreads very quickly to the rest of the population.

\subsection{Propagation time on the Poincaré disk model}

In this subsection, we consider the following metric of the space. Given $u=\left(x_{1}, y_{1}\right)$ and $v=\left(x_{2}, y_{2}\right)$ in the unit disk then we define the distance

$$
d_{\mathbb{H}}(u, v)=\operatorname{arccosh}(1+\delta(u, v)),
$$


where

$$
\delta(u, v)=2 \frac{\|u-v\|^{2}}{\left(1-\|u\|^{2}\right)\left(1-\|v\|^{2}\right)} .
$$

The values of the parameters we have used in our simulations are listed as follows:

1. The network area is the circle of radius $R$ centered at the origin.

2. To neglect border effects, we consider a "billiard" like boundary effect (where nodes bounce against the boundary in a billiard like fashion).

3. Nodes start from a uniformly distributed (in the metric sense) initial position.

4. Each node takes a uniformly distributed direction angle in $[0,2 \pi)$.

5. We evaluated different scenarios where all mobile nodes change their direction after $T_{1}=1 \mathrm{~s}, T_{2}=5 \mathrm{~s}$ and $T_{3}=10 \mathrm{~s}$.

6. Each mobile node change their speed at each new time interval and choose its speed at random with Gaussian random variable $N\left(0, v^{2}\right)$ where $v=0.005 \operatorname{diam} / \mathrm{s}$.

7. We take at random one mobile node and infect it.

8. We assume that all of the nodes within range of an infected node will be infected as well.

To make the comparisons fair we choose the number of nodes in the Euclidean and hyperbolic case to be the same per unit area. More precisely, let $n_{\mathbb{E}}$ and $n_{\mathbb{H}}$ be the number of nodes in the Euclidean and hyperbolic domain respectively we want that

$$
\frac{n_{\mathbb{H}}}{\operatorname{area}_{\mathbb{H}}(\mathbb{D}(0, R))}=n_{\mathbb{E}}
$$

Since a disk of radius $R \in[0,1]$ (with respect to the Euclidean metric) in the hyperbolic disk has hyperbolic area

$$
\operatorname{area}_{\mathbb{H}}(\mathbb{D}(0, R))=4 \pi \sinh ^{2}\left(\frac{1}{2} \log \left(\frac{1+R}{1-R}\right)\right)
$$




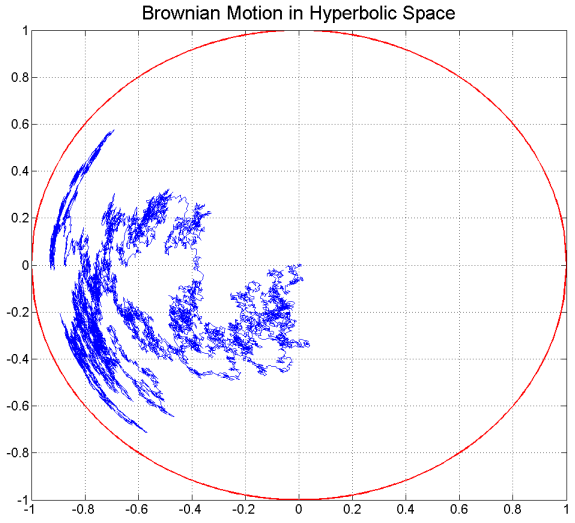

Figure 6: A node performing a Brownian motion in the Poincare disk. We show its first 15, 000 steps.

then if $n_{\mathbb{E}}=500$ and $R=0.2715$ we have that $n_{\mathbb{H}}=500$.

In Figure 6 we see a node staring from the origin and performing a Brownian motion with respect to the hyperbolic metric.

The simulation in Figure 7 shows the total propagation time, i.e., the time it takes for all of the nodes to be infected, as a function of the radius of coverage normalized by the diameter.

\subsection{Bootstrap Propagation Model on the Poincaré disk model}

As in the previous subsection, we consider the Euclidean distance to be the metric of the space. The values of the parameters we have used in our simulations are listed as follows:

The first (1)-(6) are the same as in the previous simulation.

8. We choose at random $M$ mobile nodes and infect them.

9. We assume that all of the nodes within range of an infected node will 

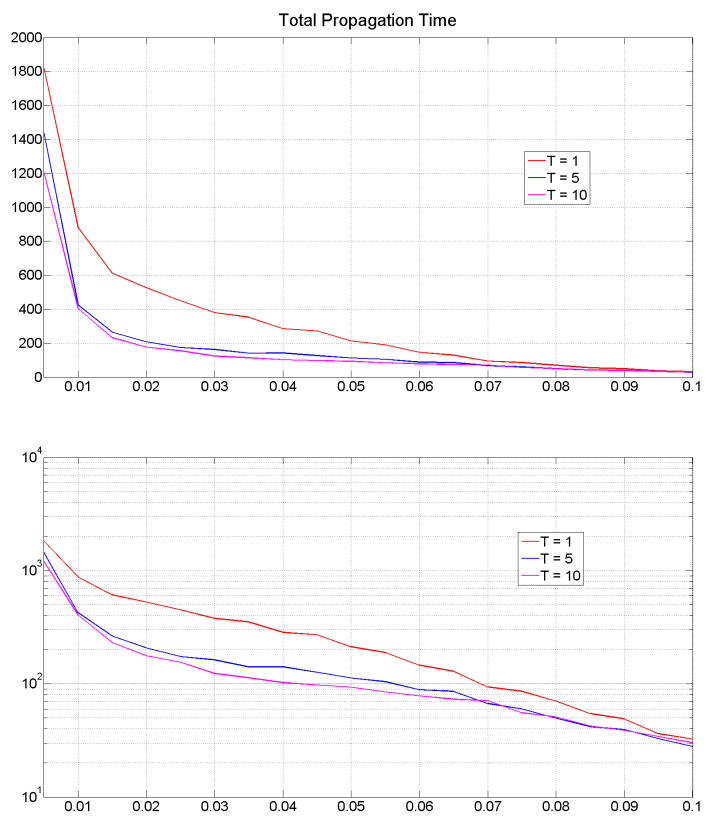

Figure 7: Total propagation time as a function of the radius of coverage normalized by the diameter for three different mobility scenarios in the hyperbolic space. The bottom figure is the log scale of the top one. 


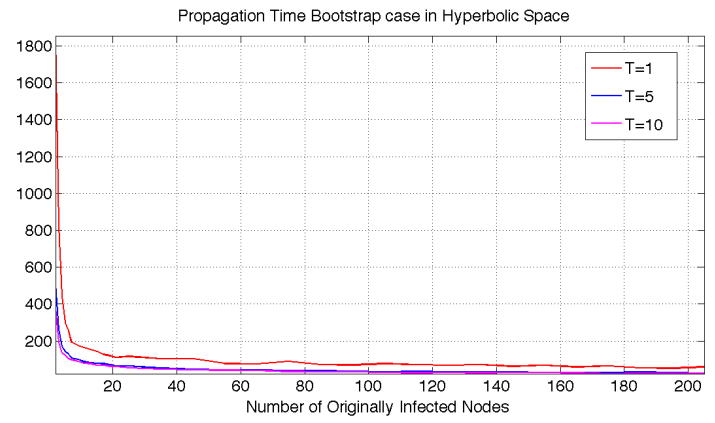

Figure 8: Total propagation time as a function of the radius of coverage for three different mobility scenarios in the bootstrap case with the hyperbolic metric.

be infected as well.

10. We consider a radius of coverage equal to $r=0.05$ diam $/ 2$.

The simulation shows the total propagation time as a function of the initial number of infected nodes $M$.

\subsection{Propagation Time in a Domain with Obstacles}

In this subsection we study the behavior of the propagation time when the domain has a certain number of obstacles. More precisely, assume that our domain is the unit square area with four small inaccessible squares inside. These small squares could model lakes or areas where the nodes are not allowed to enter. How does the propagation time is affected by these obstacles? We expect that the propagation time will increase but would it be significant?

The values of the parameters we have used in our simulations are listed as follows:

1. The network area is the two-dimensional unit square $I=[0,1] \times[0,1]$ (where nodes bounce against the boundary in a billiard like fashion) with 4 small squares removed as in Figure 9. 


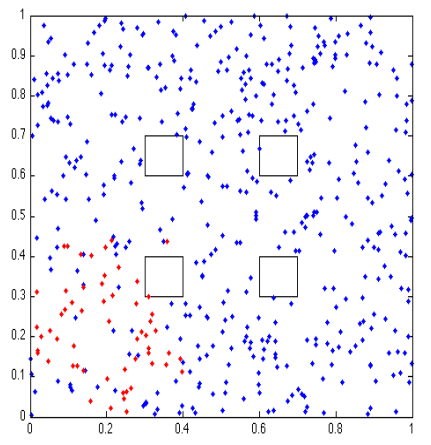

Figure 9: Infected nodes are in red and no infected ones in blue.

2. The number of mobile nodes in the network is $n=500$.

3. Nodes start from a uniformly distributed initial position.

4. Each node takes a uniformly distributed direction angle in $[0,2 \pi)$.

5. We evaluated the scenarios where all mobile nodes change their direction after $T_{1}=1 \mathrm{~s}$ and $T_{2}=5 \mathrm{~s}$.

6. Each mobile node change their speed at each new time interval and choose its speed at random with a Gaussian distribution $N\left(0, v^{2}\right)$ where $v=$ $0.005 \mathrm{diam} / \mathrm{s}$. Therefore, the standard deviation is $0.5 \%$ of the diameter of the whole domain per second.

7. We take at random one mobile node and consider it infected.

8. We assume that all of the nodes within range of an infected node will became infected.

Figure 10 shows the simulation results for the propagation time. Similar results were obtained in the bootstrap case with obstacles and in the hyperbolic case. 


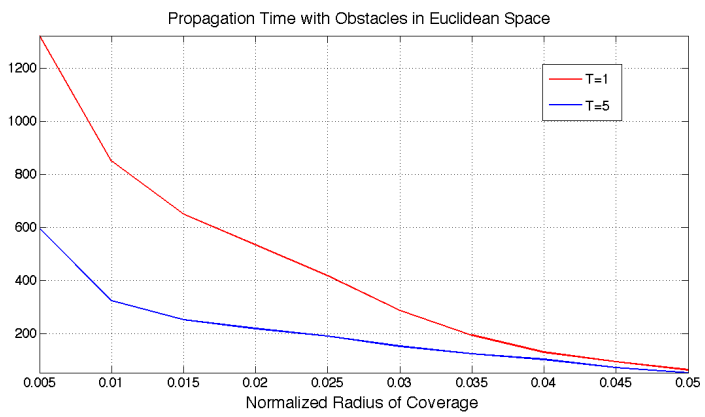

Figure 10: Total propagation time as a function of the radius of coverage normalized by the diameter for two different mobility scenarios in the Euclidean space with four obstacles.

\section{Conclusions}

A random geometric graph consists of a set of vertices randomly distributed over a metric space, with two vertices joined by an edge if the distance between them is smaller than a certain threshold called radius of coverage. In addition to the theoretical interest of these graphs, they have been useful in several applications since, while simple, these models capture important features of real world ad-hoc networks. In this work, we have studied the spectral properties of these graphs and in particular, we have proven the lack of spectral gap in random geometric graphs under a wide range of cases. We have also presented some results on the spectral properties of generalized weighted versions of these graphs.

We have later focused on mobile geometric graphs or dynamic Boolean models as were introduced by van den Berg, Meester, and White in 1997, and also on the Lévy flights since measurements suggest that animal and also human trajectories can be approximated by them $[17,18,19,20]$. These mobilility models have been useful to model mobile ad-hoc and sensor networks. Investigating the mobility at a geographical scale, is relevant for individual mobility, for the proximity of the nodes, and in consequence, for data diffusion, and its applications. In particular, on this part of our work, we focus on the study of the propagation time and how it is affected by changing the used metric and the topology of the geographical area. We call propagation 
time the time it takes for a certain message to reach all the nodes in the network. In other words, we explore how different models for the information spread affect the propagation time. In particular, we study how the metric, mobility properties, presence or absence of obstacles and finally the propagation characteristics affect the dynamics of the message spreading. Even though we could observe a change in the propagation time by changing the metric the most significant changes were observed by changing the mobility properties of the nodes and by changing how information spreads (bootstrap and non-bootstrap case). In the non-bootstrap case the propagation time seems to decay exponentially with the radius of coverage. More specifically, we could see from figures 2,4 and 7 , that the total propagation time $T$ is of the form

$$
T(r) \propto \exp (-\beta r)
$$

where $r$ is the radius of coverage of each node and $\beta$ is a parameter that depends on the mobility properties and the metric characteristics.

On the other hand, when we compute the total propagation time as a function of the initially infected nodes $m$, we observe a phase transition around $m=5$. In other words, the propagation time decays abruptly around $m=5$ and it decreases very slowly as $m$ increases. This phenomenon can be observed in figures 5 and 7 for the bootstrap case.

We did not observe any significant changes in the total propagation time by introducing obstacles in the domain.

\section{References}

[1] C. Avin and G. Ercal, On the cover time and mixing time of random geometric graphs, Theoretical Computer Science, 380:2-22, 2007.

[2] F. Chung, Spectral Graph Theory, CBMS Number 92, AMS, 1994.

[3] M. do Carmo, Riemannian Geometry, Birkhäuser Boston, 1992.

[4] R. Ellis, J. Martin and C. Yan, Random geometric graphs diameter in the unit ball, Algorithmica 47, 421-438, 2007 
[5] P. Erdös and A. Renyi, On a problem in the theory of graphs, Publ. Math. Inst. Hungar. Acad. Sci. 7, pp. 623-621, 1962.

[6] P. Erdös, A. Renyi and V. T. Sós On a problem of graph theory, Studia Sci. Math. Hungar. 1, pp. 215-235, 1966.

[7] P. Gupta and P. R. Kumar, Critical power for asymptotic connectivity in wireless networks, In W.M. McEneany, G. Yin, and Q. Zhang, editors, Stochastic Analysis, Control, Optimization and Applications: A Volume in Honor of W.H. Fleming, pages 547-566. Birkhauser, Boston, 1998.

[8] P. Gupta and P. R. Kumar, The capacity of wireless networks, IEEE Transactions on Information Theory, 46:388-404, 2000. Correction in IEEE Transactions on Information Theory 49, pp. 3117, 2000.

[9] G. Kesidis, T. Konstantopoulos and S. Phoha, Surveillance coverage of sensor networks under a random mobility strategy, In Proceedings of the 2nd IEEE International Conference on Sensors, 2003.

[10] H. Kesten and V. Sidoravicius, The spread of a rumor or infection in a moving population, The annals of probability, 33:2402-2462, 2005.

[11] B. McKay, The Expected Eigenvalue Distribution of a Large Regular Graph, Lin. Alg. and Applications, vol. 40, pp. 203-216, 1981.

[12] B. Mohar, W. Woess, A Survey on Spectra of Infinite Graphs, Bull. London Math. Soc., vol. 21, pp. 209-234, 1989.

[13] B. Mohar, The Laplacian Spectrum of Graphs, Graph Theory, Combinatorics, and Appl., vol. 2, pp. 871-898, 1991.

[14] M. Penrose, Random geometric graphs, Oxford University Press, 2003.

[15] M. Penrose, On k-connectivity for a geometric random graph, Random Structures and Algorithms, 15:145-164, 1999.

[16] Y. Peres, A. Sinclair, P. Sousi and A. Stauffer Mobile Geometric Graphs: Detection, Coverage and Percolation, preprint arXiv:1008.0075v2.

[17] J. Klafter, M. F. Shlesinger and G. Zumofen, Beyond brownian motion, Physics Today, vol. 49, 1996. 
[18] I. Rhee, M. Shin, S. Hong, K. Lee and S. Chong, On the Lévy-Walk Nature of Human Mobility, 2008 IEEE INFOCOM - The 27th Conference on Computer Communications, IEEE, pp. 924-932, 2008.

[19] S. Hong, I. Rhee, S. J. Kim, K. Lee and S. Chong, Routing Performance Analysis of Human-Driven Delay Tolerant Networks using the Truncated Lévy Walk Model, MobilityModels: Proceeding of the 1st ACM SIGMOBILE workshop on Mobility models, ACM, New York, NY, USA, pp. 25-32, 2008.

[20] M. P. Freeman, N. W. Watkins, E. Yoneki and J. Crowcroft, Rhythm and Randomness in Human Contact, 2010 International Conference on Advances in Social Networks Analysis and Mining, IEEE, pp. 184-191, 2010 .

[21] T. Camp, J. Boleng and V. Davies, A survey of mobility models for ad hoc network research, Wireless Communications and Mobile Computing, vol. 2, pp. 483-502, 2002.

[22] R. Groenevelt, P. Nain and G. Koole, The message delay in mobile ad hoc networks, Perform. Eval. 62, pp. 210-228, 2005.

[23] H. Cai and D. Y. Eun, Crossing over the bounded domain: from exponential to power-law inter-meeting time in MANET, MobiCom '07: Proceedings 21 of the 13th annual ACM international conference on Mobile computing and networking, ACM, New York, NY, USA, pp. 159-170, 2007.

[24] J. Karvo and J. Ott, Time scales and delay-tolerant routing protocols, CHANTS '08: Proceedings of the third ACM workshop on Challenged networks, ACM, New York, NY, USA, pp. 33-40, 2008.

[25] C. Boldrini, M. Conti and A. Passarella, Modelling data dissemination in opportunistic networks, CHANTS '08: Proceedings of the third ACM workshop on Challenged networks, ACM, New York, NY, USA, pp. 8996, 2008.

[26] A. Singer, From graph to manifold Laplacian: the convergence rate, Applied and Computational Harmonic Analysis, vol. 21, no. 1, pp. 128-134, 2006. 